\title{
Efficacy and safety of celecoxib on the incidence of recurrent colorectal adenomas: a systematic review and meta-analysis
}

This article was published in the following Dove Medical Press journal: Cancer Management and Research

\begin{abstract}
Sajesh K Veettil,' Surakit Nathisuwan, ${ }^{2}$ Siew Mooi Ching, ${ }^{3-5}$ Peerawat Jinatongthai, ${ }^{6,7}$ Kean Ghee Lim, ${ }^{8}$ Siang Tong Kew, ${ }^{9}$ Nathorn Chaiyakunapruk ${ }^{7,10-12}$

'Department of Pharmacy Practice, School of Pharmacy, International Medical University, Kuala Lumpur, Malaysia; ${ }^{2}$ Clinical Pharmacy Division, Department of Pharmacy, Faculty of Pharmacy, Mahidol University, Bangkok, Thailand; ${ }^{3}$ Department of Family Medicine, Faculty of Medicine and Health Sciences, Universiti Putra Malaysia, Serdang, Malaysia; ${ }^{4}$ Malaysian Research Institute on Ageing, Universiti Putra Malaysia, Serdang, Malaysia; ${ }^{5}$ Department of Medical Sciences, School of Healthcare and Medical Sciences, Sunway University, Selangor, Malaysia; ${ }^{6}$ Division of Pharmacy Practice, Faculty of Pharmaceutical Sciences, Ubon Ratchathani University, Ubon Ratchathani, Thailand; 'School of Pharmacy, Monash University Malaysia, Bandar Sunway, Selangor, Malaysia; ${ }^{8}$ Department of Surgery, Clinical School, International Medical University, Seremban, Malaysia; ' Department of Internal Medicine, School of Medicine, International Medical University, Kuala Lumpur Malaysia; ${ }^{10}$ Department of Pharmacy Practice. Faculty of Pharmaceutical Sciences, Center of Pharmaceutical Outcomes Research, Naresuan University, Phitsanulok, Thailand; "School of Pharmacy, University of Wisconsin, Madison, WI, USA; ${ }^{2}$ Asian Centre for Evidence Synthesis in Population, Implementation and Clinical Outcomes (PICO), Health and Wellbeing Cluster, Global Asia in the 21 st Century (GA2I) Platform, Monash University Malaysia, Selangor, Malaysia
\end{abstract}

Correspondence: Nathorn Chaiyakunapruk Faculty of Pharmaceutical Sciences, Naresuan University, Muang, Phitsanulok 65000, Thailand Tel +66840094460

Fax +6655963731

Email nathorn.chaiyakunapruk@monash.edu

Surakit Nathisuwan

Department of Pharmacy, Faculty of Pharmacy,

Mahidol University, 47 Sri-ayutthaya Road,

Ratchathewi, Bangkok 10400, Thailand

Tel/Fax +6626448694

Email surakit.nat@mahidol.ac.th
Background: Celecoxib has previously been shown to be effective in reducing recurrent colorectal adenomas, but its long-term effects are unknown. In addition, safety issues are of major concern. Therefore, we examined the efficacy and safety of celecoxib as a chemopreventive agent along with its posttreatment effect.

Methods: We performed a meta-analysis based on a systematic review of randomized controlled trials (RCTs) comparing celecoxib at various doses (400 mg once daily, $200 \mathrm{mg}$ twice daily, and $400 \mathrm{mg}$ twice daily) vs placebo in persons with history of colorectal adenomas. Several databases were searched from inception up to April 2018. Long-term follow-ups of RCTs were also included to evaluate posttreatment effect. Primary outcome was the incidence of recurrent colorectal adenomas. Various safety outcomes were evaluated, especially cardiovascular (CV) events. Risk-benefit integrated analyses were also performed.

Results: A total of three RCTs (4,420 patients) and three post-trial studies (2,159 patients) were included in the analysis. Use of celecoxib at any dose for 1-3 years significantly reduced the incidence of recurrent advanced adenomas (risk ratio, 0.42 [95\% CI, 0.34-0.53]) and any adenomas (0.67 [95\% CI, 0.62-0.72]) compared with placebo. Subgroup analysis on different dosing suggested a greater effect with $400 \mathrm{mg}$ twice daily. However, celecoxib $400 \mathrm{mg}$ twice daily significantly increased the risk of serious adverse (1.2 [95\% CI, 1.0-1.5]) and CV events (3.42 [95\% CI, 1.56-7.46]), while celecoxib at $400 \mathrm{mg} /$ day, especially with once daily dosing, did not increase CV risk (1.01 [95\% CI, 0.70-1.46]). Analysis of post-trial studies indicated that the treatment effect disappeared (1.15 [95\% CI, 0.88-1.49]) after discontinuing celecoxib for $>2$ years.

Conclusion: Celecoxib $400 \mathrm{mg}$ once daily dosing could potentially be considered as a viable chemopreventive option in patients with high risk of adenomas but with low CV risk. Long-term trials on celecoxib at a dose of $\leq 400 \mathrm{mg}$ either once or twice daily are warranted.

Keywords: colorectal cancer, colorectal adenomas, chemoprevention, celecoxib, meta-analysis, randomized controlled trials, risk-benefit integrated analyses

\section{Background}

Colorectal cancer (CRC) is the third most common cancer worldwide, with over 1.4 million new cases estimated to have occurred in $2012 .{ }^{1}$ It is widely accepted that adenomas/polyps are well-known precursors of sporadic CRCs. ${ }^{2}$ Early detection and removal of adenomatous polyps by colonoscopic screening has been showed to reduce mortality from $\mathrm{CRC} .{ }^{3}$ Unfortunately, surveillance colonoscopic screening is underutilized. ${ }^{4-6} \mathrm{~A}$ variety of reasons, including suboptimal adherence to screening, availability, and cost, may play a part in this problem. For those who undergo polypectomy, the 
recurrence rate is still relatively high., ${ }^{2,3,7}$ Therefore, the use of chemoprevention strategies to complement surveillance screening may have a potential to further reduce CRC morbidity and mortality among those with adenomatous polyps.

Protective effect of non-aspirin nonsteroidal antiinflammatory drugs (NSAIDs) on colorectal adenomas have been documented in previous systematic reviews. ${ }^{8-12}$ However, concerns about cardiovascular (CV) safety and risk of serious bleeding events hamper the acceptance of these strategies for secondary prevention of CRC. ${ }^{11,13}$ Cyclooxygenase-2 (COX2) inhibitors ${ }^{14}$ selectively interfere with COX-2 enzyme and have been shown to cause less major bleeding compared with traditional NSAIDs. ${ }^{15-18}$ For CV safety, most non-aspirin NSAIDs and COX-2 inhibitors have been shown to increase the risk of thrombotic CV events. ${ }^{15,19-21}$ However, the risk of these events may be a result of complex interplay among a specific drug molecule, dose, and baseline CV risk. ${ }^{16,22}$ Rofecoxib, which was withdrawn from the market, was shown to have a much higher risk compared with celecoxib. ${ }^{21}$ For celecoxib, the risk appeared to be dose dependent and was evident among patients with high CV risk at baseline. ${ }^{19}$ In addition, available evidence suggested that twice daily dosing, not once daily dosing, was associated with increased CV risk. ${ }^{19,23,24}$ Recently, celecoxib at approved doses (200-400 mg/day), was found to be noninferior to ibuprofen or naproxen with regard to $\mathrm{CV}$ safety in a large, randomized, controlled trial with over 24,000 patients. ${ }^{25}$ As a result, celecoxib at approved doses could be a viable option for patients with history of adenomas where the risk of CRC may outweigh the risk of $\mathrm{CV}$ events. ${ }^{8,26} \mathrm{We}$, therefore, conducted a systematic review and meta-analysis to evaluate efficacy and safety of celecoxib in patients with a history of adenomas. We performed risk-benefit integrated analysis to comprehensively evaluate celecoxib's multidimensional effects in this setting. Moreover, we also investigated whether the adenoma-preventive effect of celecoxib waned after withdrawal.

\section{Methods}

\section{Study design}

This study was performed as part of a systematic review that has been previously registered (PROSPERO CRD42015025849) ${ }^{27}$ and is reported according to the Preferred Reporting Items for Systematic Reviews and Meta-Analyses. ${ }^{28}$

\section{Search strategy and study selection}

We identified relevant studies through a systematic search of Medline and EMBASE until April 2018. In addition, we searched published systematic reviews for additional studies.
The search strategy is provided in Table S1. Studies included were randomized controlled trials (RCTs) and long-term follow-up of RCTs that met the following inclusion criteria: participants were adults (aged $\geq 18$ years) at an increased risk due to a previous history of adenomas who underwent polypectomy and with a documented clean colon before randomization; intervention was celecoxib at any dose; the comparator was placebo or control; and the outcome was the proportion of subjects who developed colorectal neoplasia, described as either colorectal adenomas or advanced adenomas.

\section{Outcomes of interest}

Primary efficacy outcomes of interest were the incidence of recurrent colorectal adenomas (advanced adenomas and any adenomas). Advanced adenoma was defined by one or more of the following features: $1 \mathrm{~cm}$ or larger, with villous or tubulovillous histology, with high-grade dysplasia, and/or with intramucosal carcinoma or invasive cancer. ${ }^{29}$ Any adenomas include both advanced and nonadvanced adenomas (defined as one or two small $[<1 \mathrm{~cm}]$ tubular adenomas or serrated polyps without cytologic dysplasia) ${ }^{29}$ and invasive cancers.

Safety outcomes were the incidence of CRC, mortality due to any causes, serious adverse events, serious CV events, and renal and hypertensive disorders reported on any followup after randomization. Serious adverse events were defined as events resulting in death, hospital admission because of an adverse event, severe gastrointestinal bleeding, $\mathrm{CV}$ or non-CV complications, or discontinuation of intervention due to an adverse event or events that were defined as serious or severe by study authors. Serious CV events were defined as the composite of CV death, myocardial infarction, stroke, heart failure, thromboembolic event, or defined as serious $\mathrm{CV}$ event by the study investigators. Renal and hypertensive disorders included reports of elevated serum creatinine levels, fluid retention and edema, hypertension, proteinuria, and renal failure. We also evaluated the posttreatment effects of celecoxib on the incidence of recurrent colorectal neoplasia after discontinuing the intervention for $>2$ years.

\section{Data extraction and quality assessment}

Two reviewers (SKV and KGL) screened the relevant publications and then extracted data on the study, participants, and treatment-related characteristics onto a standardized form, and discrepancies were resolved by another author after group discussion. Data on efficacy outcomes were extracted with modified intention-to-treat analysis (ie, subjects who received at least one dose of celecoxib at any dose and had 
at least one colonoscopy after randomization). Data on safety outcomes were extracted by intention-to-treat principle, using the initial number of randomized participants allocated to each trial arm. Participants who were lost to follow-up were considered free of adverse events.

Previous evidence suggested that the effects of NSAIDs on adenoma recurrence may not be sustained after treatment cessation. ${ }^{30-32}$ Hence, we abstracted efficacy outcomes measured cumulatively at two time points, within 1 year of discontinuing intervention (primary efficacy analysis) and $\geq 2$ years after discontinuing intervention (posttreatment effect analysis). Two reviewers (SKV and SMC) independently assessed the risk of bias (ROB) in the context of the primary outcome by using the revised Cochrane risk of bias tool (RoB 2.0). ${ }^{33}$

\section{Data synthesis and statistical analysis}

Meta-analysis was performed with DerSimonian and Laird random-effects model to estimate pooled risk ratios and 95\% confidence intervals incorporating heterogeneity within and between studies, with Stata version 14.0 (StataCorp, College Station, TX, USA). ${ }^{34}$ Statistical heterogeneity between trials was assessed for primary outcomes using $I^{2}$ statistics, with values $>50 \%$ indicating substantial levels of heterogeneity. ${ }^{35,36}$ Publication bias could not be assessed due to the small number of included studies, which limited the power to distinguish between finding by chance and real asymmetry. ${ }^{37}$ Subgroup analyses were performed for different dosings of celecoxib ${ }^{38}$ including $200 \mathrm{mg}$ twice daily (400 mg/day), $400 \mathrm{mg}$ once daily, and $400 \mathrm{mg}$ twice daily (800 mg/day). Sensitivity analyses were performed based on the use of surveillance colonoscopy per protocol completer analysis (outcomes included only those subjects who underwent colonoscopy surveillance at the prespecified time period per protocol and excluded subjects who underwent a colonoscopic surveillance assessment before the expected surveillance interval), fixed-effect model, and trials with low ROB.

The Grading of Recommendations, Assessment, Development and Evaluation (GRADE) approach was used to rate the quality of evidence (high, moderate, low, and very low) of estimates derived from meta-analyses using GRADEpro version 3.6.1 (McMaster University, 2014). ${ }^{39}$

\section{Risk-benefit integrated analysis}

Similar to approaches used in previous meta-analysis, ${ }^{8}$ we used risk-benefit integrated analysis to review the potential benefits (prevention of recurrent advanced adenomas) and risks (serious adverse events and CV events) of celecoxib at
400 and $800 \mathrm{mg} /$ day. To understand potential benefits at the population level, we used risk ratios derived from the placebo comparisons of celecoxib in the meta-analysis to estimate absolute risk of advanced adenomas with intervention. We used published pooled estimates from the National Cancer Institute pooling project to estimate population-level risks of advanced adenomas as assumed control risk (7.4\% in low-risk group or population with history of nonadvanced adenomas and $16.3 \%$ in high-risk group or population with history of advanced adenomas). ${ }^{40}$ Similarly, to understand the potential risks, we used the pooled risk of serious adverse events in placebo groups in the meta-analysis (estimated using metaprop command in STATA) as a measure of baseline risk. We then used risk ratios derived from the placebo comparisons of celecoxib in the meta-analyses for serious adverse events and $\mathrm{CV}$ events to estimate absolute risk associated with celecoxib. We then presented excess benefit and risk of serious adverse events (over placebo) per 1,000 individuals who received treatment. Estimates of absolute risk were generated with the GRADEpro version 3.6.1 (McMaster University, 2014). ${ }^{39}$

\section{Results Study selection}

We identified 391 records in which 11 potentially eligible articles were reviewed in full text. Of these, six articles were excluded mostly due to the lack of eligible population. Therefore, a total of five studies were included in our review. Among these five studies, three trials ${ }^{23,24,41}$ met the eligibility criteria for the quantitative analysis of primary outcomes. Post-trial results from three studies ${ }^{30,31,41}$ were included for the analysis of posttreatment effect on the incidence of recurrent adenomas. The PRISMA flow diagram depicting the search and selection process for the primary outcomes is displayed in Figure S1.

\section{Characteristics of the included studies}

Table 1 describes the characteristics of three RCTs ${ }^{23,24,41}$ which reported the incidence of recurrent colorectal adenomas. A total of 4,420 participants with a previous history of adenomas who underwent polypectomy and with documented clean colon before randomization were included in the analysis. All trials were double-blinded and placebo-controlled. The treatment duration was 3 years in two trials $\mathrm{s}^{23,24}$ and 1 year in one trial. ${ }^{41}$ Postrandomization colonoscopy was performed within 1 year of discontinuing intervention in all trials. The dose per day of celecoxib used in two trials was $400 \mathrm{mg}$ once daily, ${ }^{23,41}$ and the remaining trial ${ }^{24}$ tested both $400 \mathrm{mg}$ (200 $\mathrm{mg}$ twice daily) and $800 \mathrm{mg}$ (400 mg twice daily) doses. A 


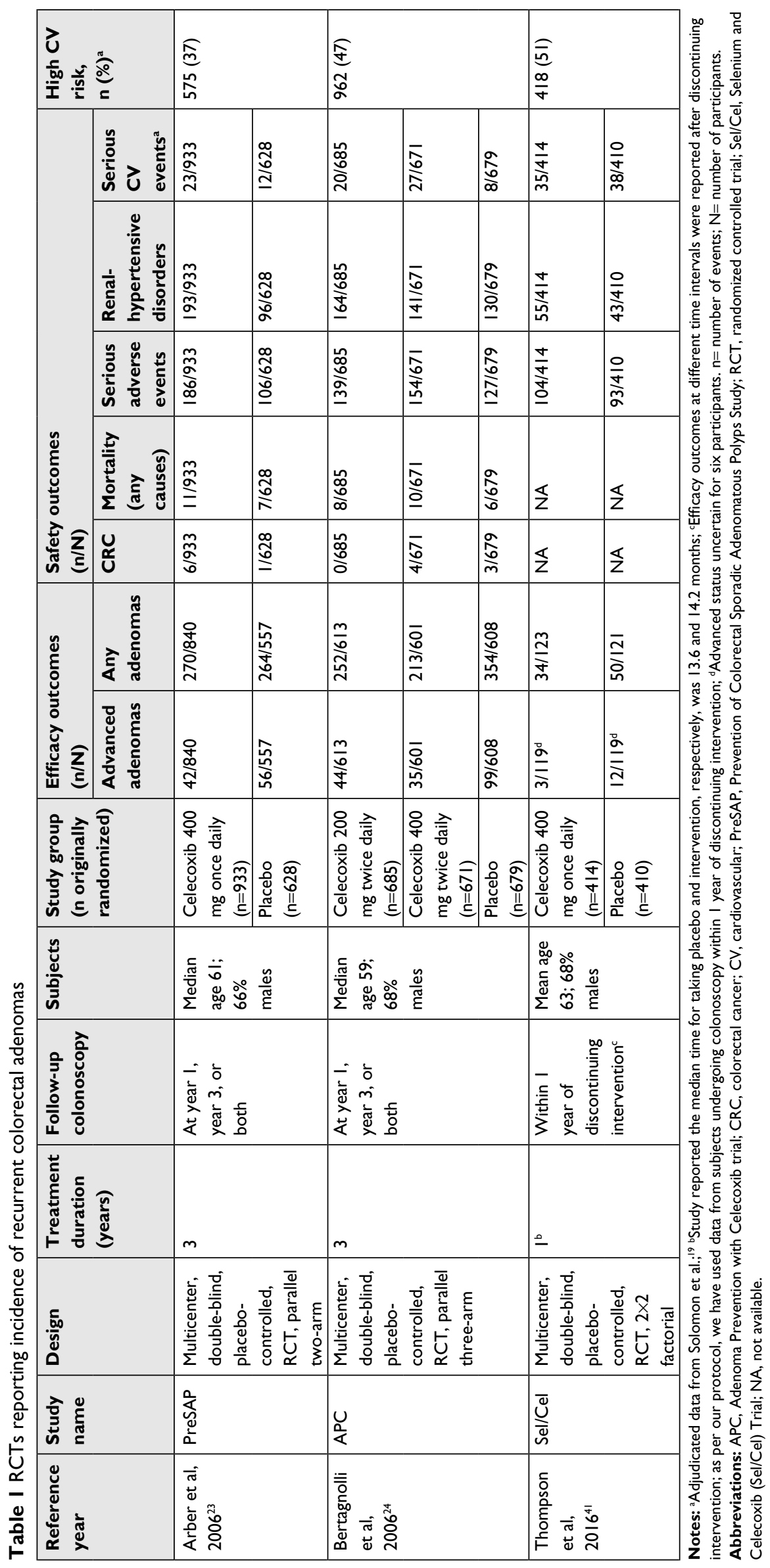


detailed description of ROB assessment among included RCTs is presented in Table S2. Among three RCTs, one trial ${ }^{41}$ was judged to be at high ROB and the remaining two trials $\mathrm{s}^{23,24}$ were judged to be at low ROB in all domains.

Post-trial results from three studies ${ }^{30,31,41}$ were available to investigate the effect of celecoxib withdrawal on incidence of recurrent colorectal adenomas. Table 2 describes the identified studies. A total of 2,159 participants who completed follow-up colonoscopy after discontinuing intervention for 2-4 years were included in the analysis.

\section{Effects on the primary efficacy outcomes}

Based on the results from the meta-analyses, use of celecoxib at any dose (400-800 $\mathrm{mg} /$ day) for a duration of approximately 1-3 years showed a statistically significant reduction in the recurrence of advanced colorectal adenomas (RR, 0.42 [95\% CI, 0.34-0.53]) and any adenomas (RR, 0.67 [95\% CI, 0.62-0.72]) compared with placebo, with no heterogeneity $\left(I^{2}=0 \%\right.$; Figure 1$)$. In the sensitivity analyses (Figures S2-S4), findings were robust and consistent with the primary analysis. A subgroup analysis of celecoxib at 400 $\mathrm{mg}$ /day demonstrated similar effects on advanced adenomas (RR, 0.45 [95\% CI, 0.35-0.58]) and any adenomas (RR, 0.69 [95\% CI, 0.64-0.75]), with no heterogeneity ( $I^{2}=0 \%$; Figure 1). Both $400 \mathrm{mg}$ once daily and $200 \mathrm{mg}$ twice daily dosing regimens provided similar effect size (Figure S5). For celecoxib at $800 \mathrm{mg} /$ day, RR for advanced adenomas was 0.34 [95\% CI, 0.24-0.50] and 0.55 [95\% CI, 0.48-0.64] for any adenomas.

\section{Effects on the safety outcomes}

Results from meta-analyses (Figure 2) showed that celecoxib at any dose for a duration of approximately $1-3$ years significantly increases the risk of serious adverse events (RR, 1.15 [95\% CI, 1.02-1.30]) and renal-hypertensive disorders (RR, 1.25 [95\% CI, 1.09-1.42]) compared with placebo. Other safety outcomes including serious CV events (RR, 1.44 [95\% CI, 0.73-2.84]), all-cause mortality (RR, 1.27 [95\% CI, 0.66-2.44]), and CRCs (RR, 1.41 [95\% CI, 0.24-8.27]) were not significantly increased compared to placebo. Subgroup analysis based on different dosing regimens showed that celecoxib $400 \mathrm{mg}$ twice daily ( $800 \mathrm{mg} /$ day) significantly increased the risk of serious adverse (RR, 1.2 [95\% CI, 1.0-1.5]) and CV events (RR, 3.42 [95\% CI, 1.56-7.46]). Interestingly, for celecoxib at $400 \mathrm{mg}$ / day, a significant increase in $\mathrm{CV}$ risk was observed only with $200 \mathrm{mg}$ twice daily regimen (RR, 2.48 [95\% CI, 1.10-5.59]) but not with $400 \mathrm{mg}$ once daily regimen (RR, 1.01 [95\% CI, 0.70-1.46]), compared with placebo (Figures S6 and S7).

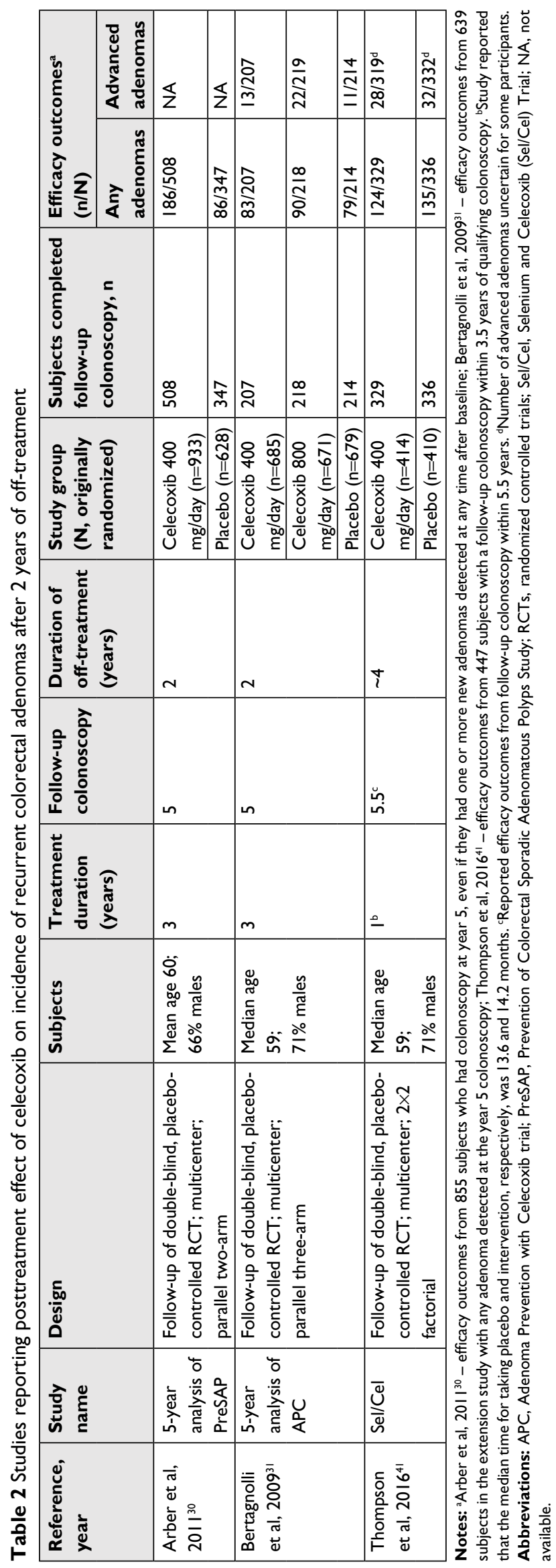




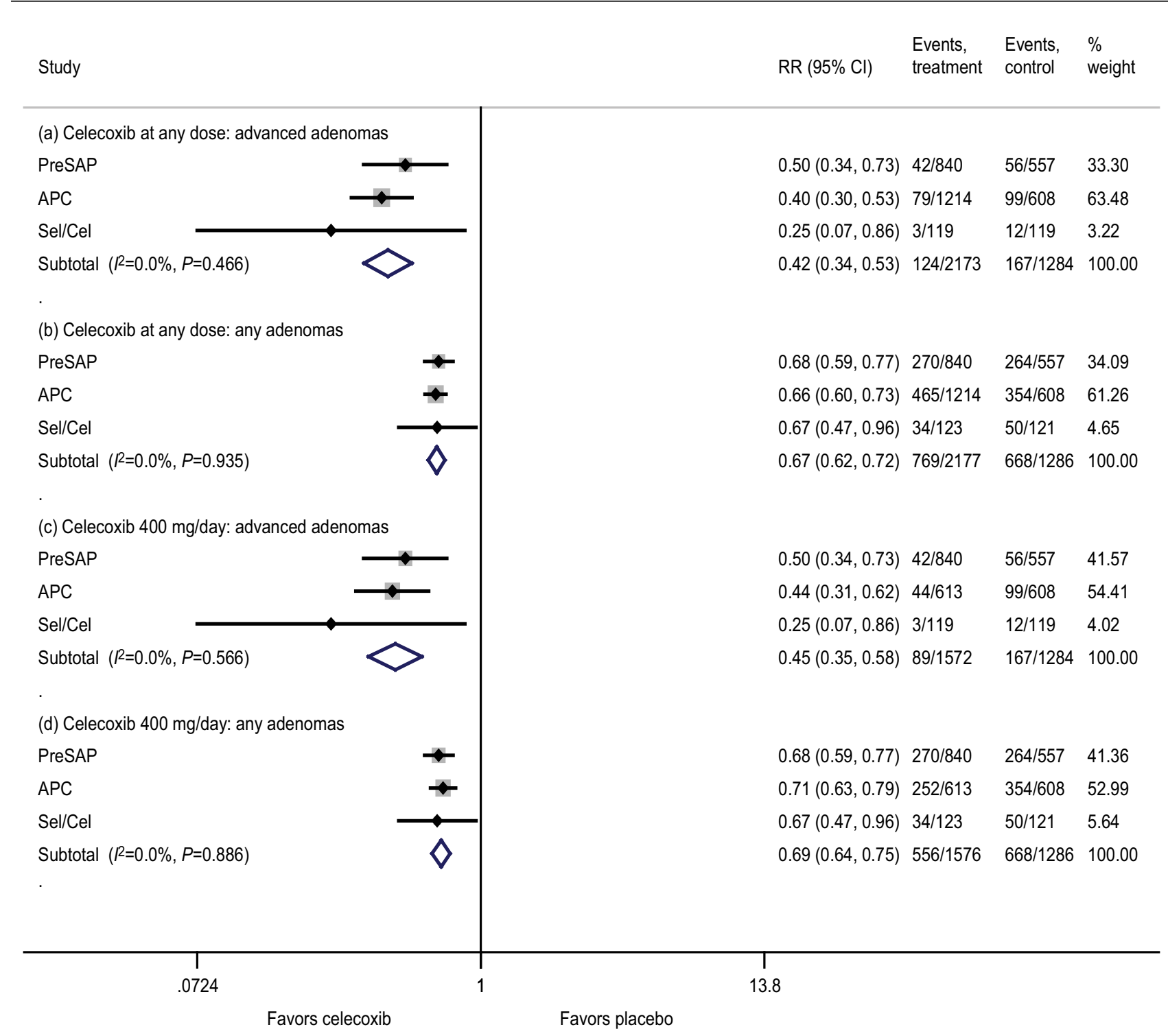

Figure I Effects on the primary efficacy outcomes.

Notes: Efficacy outcomes measured cumulatively from baseline, on postrandomization colonoscopy performed within I year of discontinuing intervention. Celecoxib at any dose: $400-800 \mathrm{mg} /$ day.

Abbreviations: APC, Adenoma Prevention with Celecoxib trial; PreSAP, Prevention of Colorectal Sporadic Adenomatous Polyps study; Sel/Cel, Selenium and Celecoxib (Sel/Cel) Trial.

\section{Grade summary of evidence}

Our application of GRADE methodology led us to conclude that the accumulated evidence for celecoxib (at any dose and $400 \mathrm{mg}$ /day) is of high quality for adenoma prevention. Detailed information on GRADE summary of evidence is presented in Table S3.

\section{Risk-benefit integrated analysis}

Based on integrated analysis, we estimated that the use of celecoxib at $800 \mathrm{mg} /$ day compared with placebo may lead to 108 (95\% CI, 82-124) and 49 (95\% CI, 30-56) fewer advanced adenomas in 1,000 patients with high-risk and low-risk adenomas, respectively. On the other hand, this would lead to an excess of $38(95 \% \mathrm{CI}, 0-95)$ serious adverse events and 82 (95\% CI, 19-186) CV events compared with placebo. As for celecoxib at $400 \mathrm{mg} /$ day, there would be 90 (95\% CI, 68-106) and 41 (95\% CI, 32-48) fewer advanced adenomas in persons with high-risk and low-risk adenomas, respectively. As for harm, this intervention would lead to an excess of 25 (95\% CI, 2 fewer to 53 more) serious adverse events and 11 ( $95 \%$ CI, 8 fewer to 44 more) CV events, per 1,000 patients treated compared with placebo. The riskbenefit balance may also be different when different dosing regimens of $400 \mathrm{mg} /$ day are used. Interestingly, celecoxib at $400 \mathrm{mg}$ once daily may be associated with less harm since this dosing regimen would only lead to 30 excess harm- 


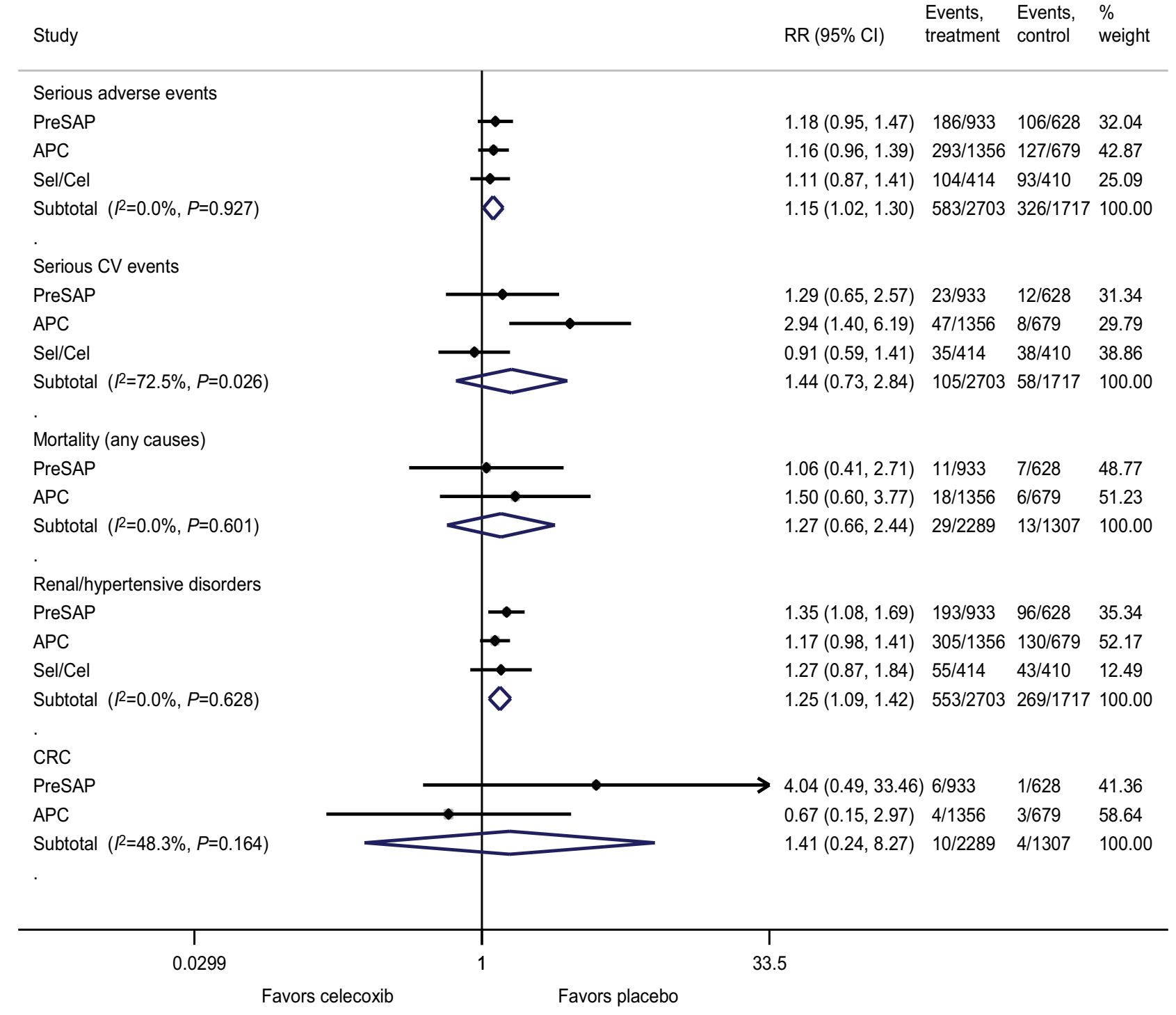

Figure 2 Effects of celecoxib at any dose on the safety outcomes.

Abbreviations: APC, Adenoma Prevention with Celecoxib trial; CRC, colorectal cancer; CV, cardiovascular; PreSAP, Prevention of Colorectal Sporadic Adenomatous Polyps Study; Sel/Cel, Selenium and Celecoxib (Sel/Cel) Trial.

ful events (29 serious adverse events and one CV event) compared with 65 events ( 15 serious adverse events and 50 CV events) with $200 \mathrm{mg}$ twice daily regimen. Based on this analysis, the risk-benefit balance of celecoxib at $400 \mathrm{mg} /$ day, especially with $400 \mathrm{mg}$ once daily dosing may be acceptable especially among patients at high risk of CRC and at low CV risk (Table S4).

\section{Posttreatment effect on efficacy outcomes}

Meta-analyses of post-trial studies demonstrated no effect on the recurrence of advanced colorectal adenomas (RR, 1.15 [95\% CI, 0.67-1.99]) and any adenomas (RR, 1.15 [95\% CI, 0.88-1.49]) after discontinuing celecoxib for more than
2 years (Figure $\mathrm{S} 8$ ). There was moderate to substantial level of heterogeneity observed in both analyses.

\section{Discussion}

Previous meta-analyses suggested a substantial protective effect of non-aspirin NSAIDs on colorectal adenoma recurrence. ${ }^{8-12}$ Although the exact mechanism of action remains to be elucidated, both COX-dependent and COX-independent mechanisms have been shown to contribute to the antitumor effects. Despite such promising data, use of long-term NSAIDs for cancer prevention is not very well received due to their significant toxicity, especially CV toxicity. ${ }^{8,11}$ However, $\mathrm{CV}$ toxicity of NSAIDs is a complicated phenomenon, which is a result of a complex interplay mainly between baseline 
$\mathrm{CV}$ risk and the nature of each NSAID along with its dose. Celecoxib, a selective COX-2 inhibitor, is among a few NSAIDs that have been tested in a number of studies for CRC prevention. Although its efficacy in this indication looked initially promising, CV toxicity shown from cancer prevention trials thwarted the interest of the medical community.

Nevertheless, a previous review has suggested that moderate dose of celecoxib ( $\leq 400 \mathrm{mg} /$ day) might not be associated with an increased CV risks. ${ }^{26}$ Recently, the PRECISION trial ${ }^{25}$ which was a large, randomized, CV safety trial comparing celecoxib at $\leq 400 \mathrm{mg} /$ day with ibuprofen and naproxen suggested that celecoxib at this dose may not confer unacceptable $\mathrm{CV}$ risk along with other serious adverse events. As a result, a comprehensive evaluation to understand the totality of its risk-benefit in this important indication may be warranted. Our study was designed to shed some light into this important question. To the best of our knowledge, this study combined the entire body of relative and absolute efficacy and safety of celecoxib at different doses. We also tried to gage the magnitude of risk and benefit in patients with varying risk of adenomas to increase understanding of the risk-benefit equation, which may vary with different levels of risk. Thus, a clearer picture of an agent with multidimensional effects can be seen.

The results of our study showed that celecoxib demonstrated a dose-dependent effect in the reduction of adenoma recurrence and the risks of serious adverse events during a follow-up of up to 3 years. Our findings on celecoxib are consistent with those from previous meta-analyses ${ }^{8-10}$ regarding the benefits of non-aspirin NSAIDs for the prevention of recurrent adenomas. For risk-benefit balance, we found that benefit of celecoxib at $400 \mathrm{mg} /$ day may outweigh the risks of adverse events in patients with high-risk adenomas. It is important to note that the absolute risk of serious $\mathrm{CV}$ events associated with NSAIDs was greatest among individuals with high CV risk at baseline. ${ }^{13,26,42}$ Our analysis demonstrated no significant risks of CV events in patients on celecoxib $400 \mathrm{mg} /$ day for 1-3 years despite that $45 \%$ of the study population were at high $\mathrm{CV}$ risk. This finding is consistent with the previous metaanalysis of randomized trials which reported no significant increase in CV risk with celecoxib $400 \mathrm{mg} /$ day. ${ }^{19,43}$ Data from other observational studies also support such findings. ${ }^{26}$ As a result, celecoxib $400 \mathrm{mg} /$ day could potentially be considered as a viable chemopreventive agent in patients with high risk of adenomas but with low CV risk. In addition, subgroup analysis based on different dosing regimens suggested that celecoxib $400 \mathrm{mg}$ once daily may have a much better safety profile on CV events compared with $200 \mathrm{mg}$ twice daily. The finding that twice daily dosing may confer greater CV risk than once daily dosing of celecoxib in our study is consistent with previous meta-analysis along with other mechanistic studies. ${ }^{19}$ Twice daily dosing of celecoxib has been shown to increase blood pressure more than that in once daily dosing. ${ }^{44} \mathrm{~A}$ previous pharmacodynamic study conducted in healthy volunteers suggested that prostacyclin levels can recover to normal levels 12 hours after a single daily dose of celecoxib. ${ }^{45}$ This raised a possibility of a more complete prostacyclin inhibition with twice daily than once daily dosing which ultimately leads to higher CV risk. Several other hypotheses have been proposed to explain differences in types and dosing of NSAIDs vs CV risk including different levels of inhibition of endothelial NO synthase ${ }^{46}$ and differential effects on the enhancement of methylarginines formation. ${ }^{47}$ However, the exact mechanism of this difference remains unknown.

For high-dose celecoxib ( $800 \mathrm{mg} /$ day), despite yielding slightly higher efficacy (18 more cases of advanced adenoma prevented compared with $400 \mathrm{mg} /$ day), the overall risk was most likely unacceptable. With 108 advanced adenomas prevented, the trade-off was 82 excess $\mathrm{CV}$ events per 1,000 patients treated with $800 \mathrm{mg} /$ day of celecoxib. This finding is very much consistent with previous meta-analysis, RCTs, and observational studies which indicated a high risk of $\mathrm{CV}$ events with a high dose of celecoxib. As a result, celecoxib at $800 \mathrm{mg} /$ day is clearly not a viable option for this indication. Of interest is the fact that no trial has ever been conducted to evaluate celecoxib at a dose of 100-200 mg/day as a chemopreventive agent. Although the efficacy of this low dose is uncertain, adverse effects of celecoxib at $\leq 200 \mathrm{mg}$ /day would most likely be less than $400 \mathrm{mg}$ /day. This may allow celecoxib to be more acceptable for long-term use if it can be demonstrated to show some efficacy for this indication at this low level of dosing.

The preventive effect of celecoxib, as shown in our analysis, waned after $\geq 2$ years of treatment cessation. The lack of a sustained clinical effect may reflect a rebound of COX-2 expression, or cessation of an alternative mechanism independent of COX-2 inhibition, as described previously. ${ }^{30,48}$ This is consistent with an increased risk of adenomas after 2 years of celecoxib discontinuation in the PreSAP trial, ${ }^{30}$ which is similar to the APPROVe study with rofecoxib. ${ }^{32}$ These data suggested that celecoxib may need to be employed on a long-term basis. Since both efficacy and adverse events can be cumulative, future study of celecoxib may need to be long term to fully elucidate the true balance on risk and benefit of this agent. 
Our study has several important limitations. First, the limited number of trials along with their short duration, some of which were terminated early, may not provide sufficient data to genuinely represent the long-term risk and benefit of celecoxib. Second, by using a pooled estimate of population data from the National Cancer Institute pooling project, our analysis may be limited by the nature of the source data that were derived mainly from a Caucasian population and a specific geographical area. Since significant differences exist among various subtypes of CRC across the world, the applicability of such data to other parts of the world may be limited. This is also the case for pooled estimate on $\mathrm{CV}$ events where different rates of $\mathrm{CV}$ events are seen among different racial groups and geographical areas. Third, we did not analyze the impact of aspirin use on the risk-benefit of celecoxib. This was due to the fact that we were unable to obtain patient-level data of these included trials. Aspirin could potentially alter the risk-benefit balance of celecoxib if used concomitantly. This is due to the fact that aspirin has a modest protective effect on CRC and CV events, yet possesses gastrointestinal toxicity along with major bleedings. ${ }^{10,49,50}$ As a result, readers should be aware of this limitation. Lastly, based on these limitations, our data are useful only for hypothesis generation and cannot be considered definitive. Future research on celecoxib as a chemopreventive agent may potentially be considered but must be employed at a dose of $\leq 400 \mathrm{mg}$ either once or twice daily.

\section{Conclusion}

In this comprehensive evaluation with risk-benefit integrated analysis, celecoxib at the dose of $\leq 400 \mathrm{mg}$ /day could potentially be considered as a viable chemopreventive option, especially with a $400 \mathrm{mg}$ once daily regimen. This may be particularly attractive in patients with high risk of adenomas but with low CV risk. Example of this patient group is a patient who has a history of high-risk adenomas and also has $<5 \%$ of a 10 -year risk of developing atherosclerotic CV disease. Celecoxib at a higher dose should be discouraged due to the unacceptable high level of risk compared with small benefit gained by increasing the dose beyond $400 \mathrm{mg} /$ day. However, more long-term trials on celecoxib at a dose of $\leq 400 \mathrm{mg}$ either once or twice daily are warranted to fully elucidate the true balance on risk and benefit of this agent.

\section{Transparency declaration}

The corresponding authors affirm that the manuscript is an honest, accurate, and transparent account of the study being reported, that no important aspects of the study have been omitted, and that any discrepancies from the study as planned have been explained.

\section{Data sharing statement}

Technical appendix and dataset are available from the corresponding author.

\section{Acknowledgments}

The authors wish to thank Professor Brian L Furman, Strathclyde Institute of Pharmacy and Biomedical Sciences, Glasgow, UK, for his valuable comments and support which helped to improve the manuscript, and Mr Razman Shah Mohd Razali, reference librarian, International Medical University, for providing the full-text articles whenever needed.

\section{Disclosure}

The authors report no conflicts of interest in this work.

\section{References}

1. Ferlay J, Soerjomataram I, Dikshit R, et al. Cancer incidence and mortality worldwide: sources, methods and major patterns in GLOBOCAN 2012. Int J Cancer. 2015;136(5):E359-E386.

2. Strum WB. Colorectal adenomas. NEnglJMed.2016;374(11):1065-1075.

3. Lieberman DA, Rex DK, Winawer SJ, Giardiello FM, Johnson DA, Levin TR. Guidelines for colonoscopy surveillance after screening and polypectomy: a consensus update by the US Multi-Society Task Force on Colorectal Cancer. Gastroenterology. 2012;143(3):844-857.

4. Hoover S, Subramanian S, Tangka FKL, et al. Patients and caregivers costs for colonoscopy-based colorectal cancer screening: experience of low-income individuals undergoing free colonoscopies. Eval Program Plann. 2017;62:81-86.

5. Subramanian S, Bobashev G, Morris RJ. When budgets are tight, there are better options than colonoscopies for colorectal cancer screening. Health Aff. 2010;29(9):1734-1740.

6. Ng SC, Wong SH. Colorectal cancer screening in Asia. Br Med Bull. 2013;105:29-42.

7. Cottet V, Jooste V, Fournel I, Bouvier AM, Faivre J, Bonithon-Kopp C. Long-term risk of colorectal cancer after adenoma removal: a population-based cohort study. Gut. 2012;61(8):1180-1186.

8. Dulai PS, Singh S, Marquez E, et al. Chemoprevention of colorectal cancer in individuals with previous colorectal neoplasia: systematic review and network meta-analysis. BMJ. 2016;355:i6188.

9. Veettil SK, Teerawattanapong N, Ching SM, et al. Effects of chemopreventive agents on the incidence of recurrent colorectal adenomas: a systematic review with network meta-analysis of randomized controlled trials. Onco Targets Ther. 2017;10:2689-2700.

10. Veettil SK, Lim KG, Ching SM, Saokaew S, Phisalprapa P, Chaiyakunapruk N. Effects of aspirin and non-aspirin nonsteroidal antiinflammatory drugs on the incidence of recurrent colorectal adenomas: a systematic review with meta-analysis and trial sequential analysis of randomized clinical trials. BMC Cancer. 2017;17(1):763.

11. Rostom A, Dubé C, Lewin G, et al. Nonsteroidal anti-inflammatory drugs and cyclooxygenase- 2 inhibitors for primary prevention of colorectal cancer: a systematic review prepared for the U.S. Preventive Services Task Force. Ann Intern Med. 2007;146(5):376-389.

12. Dubé C, Rostom A, Lewin G, et al. The use of aspirin for primary prevention of colorectal cancer: a systematic review prepared for the U.S. Preventive Services Task Force. Ann Intern Med. 2007;146(5): 365-375. 
13. Bello AE, Holt RJ. Cardiovascular risk with non-steroidal anti-inflammatory drugs: clinical implications. Drug Saf. 2014;37(11):897-902.

14. Bresalier RS, Sandler RS, Quan H, et al. Cardiovascular events associated with rofecoxib in a colorectal adenoma chemoprevention trial. $N$ Engl J Med. 2005;352(11):1092-1102.

15. Bombardier C, Laine L, Reicin A, et al. Comparison of upper gastrointestinal toxicity of rofecoxib and naproxen in patients with rheumatoid arthritis. VIGOR Study Group. N Engl J Med. 2000;343(21):1520-1528.

16. Silverstein FE, Faich G, Goldstein JL, et al. Gastrointestinal toxicity with celecoxib vs nonsteroidal anti-inflammatory drugs for osteoarthritis and rheumatoid arthritis: the CLASS study: a randomized controlled trial. Celecoxib Long-term Arthritis Safety Study. JAMA. 2000;284(10):1247-1255.

17. Chan FKL, Lanas A, Scheiman J, Berger MF, Nguyen H, Goldstein JL. Celecoxib versus omeprazole and diclofenac in patients with osteoarthritis and rheumatoid arthritis (CONDOR): a randomised trial. Lancet. 2010;376(9736):173-179.

18. Aschenbrenner DS. Cardiovascular risk of celecoxib no worse than that of ibuprofen or naproxen. Am J Nurs. 2018;118(10):19.

19. Solomon SD, Wittes J, Finn PV, et al. Cardiovascular risk of celecoxib in 6 randomized placebo-controlled trials: the cross trial safety analysis. Circulation. 2008;117(16):2104-2113.

20. Kearney PM, Baigent C, Godwin J, Halls H, Emberson JR, Patrono C. Do selective cyclo-oxygenase-2 inhibitors and traditional non-steroidal anti-inflammatory drugs increase the risk of atherothrombosis? Metaanalysis of randomised trials. BMJ. 2006;332(7553):1302-1308.

21. Graham D, Campen D, Hui R, et al. Risk of acute myocardial infarction and sudden cardiac death in patients treated with cyclo-oxygenase 2 selective and non-selective non-steroidal anti-inflammatory drugs: nested case-control study. Lancet. 2005;365(9458):475-481.

22. Meek IL, Mart AFJ van de Laar, Vonkeman HE. Non-steroidal antiinflammatory drugs: an overview of cardiovascular risks. Pharmaceuticals. 2010;3(7):2146-2162.

23. Arber N, Eagle CJ, Spicak J, et al. Celecoxib for the prevention of colorectal adenomatous polyps. N Engl J Med. 2006;355(9):885-895.

24. Bertagnolli MM, Eagle CJ, Zauber AG, et al. Celecoxib for the prevention of sporadic colorectal adenomas. NEngl J Med. 2006;355(9):873-884.

25. Nissen SE, Yeomans ND, Solomon DH, et al. Cardiovascular safety of celecoxib, naproxen, or ibuprofen for arthritis. $N$ Engl J Med. 2016;375(26):2519-2529.

26. Mcgettigan P, Henry D. Cardiovascular risk and inhibition of cyclooxygenase: a systematic review of the observational studies of selective and nonselective inhibitors of cyclooxygenase2. JAMA. 2006;296(13):1633-1644.

27. Veettil SK, Saokaew S, Lim KG, Ching SM, Phisalprapa P, Chaiyakunapruk N. Comparative effectiveness of chemopreventive interventions for colorectal cancer: protocol for a systematic review and network meta-analysis of randomised controlled trials. J Gastrointest Oncol. 2016;7(4):595-602.

28. Moher D, Liberati A, Tetzlaff J, Altman DG, PRISMA Group. Preferred reporting items for systematic reviews and meta-analyses: the PRISMA statement. Ann Intern Med. 2009;151(4):W64:264-269.

29. Short MW, Layton MC, Teer BN, Domagalski JE. Colorectal cancer screening and surveillance. Am Fam Physician. 2015;91(2):93-100.

30. Arber N, Spicak J, Rácz I, et al. Five-year analysis of the prevention of colorectal sporadic adenomatous polyps trial. Am J Gastroenterol. 2011;106(6):1135-1146.

31. Bertagnolli MM, Eagle CJ, Zauber AG, et al. Five-year efficacy and safety analysis of the Adenoma Prevention with Celecoxib Trial. Cancer Prev Res. 2009;2(4):310-321.
32. Baron JA, Sandler RS, Bresalier RS, et al. A randomized trial of rofecoxib for the chemoprevention of colorectal adenomas. Gastroenterology. 2006;131(6):1674-1682.

33. Higgins JPT, Sterne JAC, Savovic J, et al. A revised tool for assessing risk of bias in randomized trials. Cochrane Database Syst Rev. 2016;10(Suppl 1):3.

34. Dersimonian R, Laird N. Meta-analysis in clinical trials revisited. Contemp Clin Trials. 2015;45(Pt A):139-145.

35. Higgins JP, Thompson SG, Deeks JJ, Altman DG. Measuring inconsistency in meta-analyses. BMJ. 2003;327(7414):557-560.

36. Melsen WG, Bootsma MC, Rovers MM, Bonten MJ. The effects of clinical and statistical heterogeneity on the predictive values of results from meta-analyses. Clin Microbiol Infect. 2014;20(2):123-129.

37. Sterne JA, Sutton AJ, Ioannidis JP, et al. Recommendations for examining and interpreting funnel plot asymmetry in meta-analyses of randomised controlled trials. BMJ. 2011;343:d4002.

38. Antman EM, Bennett JS, Daugherty A, et al. Use of nonsteroidal antiinflammatory drugs: an update for clinicians: a scientific statement from the American Heart Association. Circulation. 2007;115(12): 1634-1642.

39. Brozek JL, Alk EA, Alonso-Coello P, et al. Grading quality of evidence and strength of recommendations in clinical practice guidelines. Part 1 of 3 . An overview of the GRADE approach and grading quality of evidence about interventions. Allergy. 2009;64(5):669-677.

40. Martínez ME, Baron JA, Lieberman DA, et al. A pooled analysis of advanced colorectal neoplasia diagnoses after colonoscopic polypectomy. Gastroenterology. 2009;136(3):832-841.

41. Thompson PA, Ashbeck EL, Roe DJ, et al. Celecoxib for the prevention of colorectal adenomas: results of a suspended randomized controlled trial. J Natl Cancer Inst. 2016;108(12):djw151.

42. Coxib and traditional NSAID Trialists' (CNT) Collaboration. Vascular and upper gastrointestinal effects of non-steroidal anti-inflammatory drugs: meta-analyses of individual participant data from randomised trials. Lancet. 2013;382(9894):769-779.

43. Gunter BR, Butler KA, Wallace RL, Smith SM, Harirforoosh S. Nonsteroidal anti-inflammatory drug-induced cardiovascular adverse events: a meta-analysis. J Clin Pharm Ther. 2017;42(1):27-38.

44. Solomon SD, Pfeffer MA, Mcmurray JJV, et al. Effect of celecoxib on cardiovascular events and blood pressure in two trials for the prevention of colorectal adenomas. Circulation. 2006;114(10):1028-1035.

45. Grosser T, Fries S, Fitzgerald GA. Biological basis for the cardiovascular consequences of COX-2 inhibition: therapeutic challenges and opportunities. J Clin Invest. 2006;116(1):4-15.

46. Yu Y, Ricciotti E, Scalia R, et al. Vascular COX-2 modulates blood pressure and thrombosis in mice. Sci Transl Med. 2012;4(132):132ra54.

47. Grosser T, Ricciotti E, Fitzgerald GA. The cardiovascular pharmacology of nonsteroidal anti-inflammatory drugs. Trends Pharmacol Sci. 2017;38(8):733-748

48. Hanif R, Pittas A, Feng Y, et al. Effects of nonsteroidal anti-inflammatory drugs on proliferation and on induction of apoptosis in colon cancer cells by a prostaglandin-independent pathway. Biochem Pharmacol. 1996;52(2):237-245.

49. Bibbins-Domingo K. Aspirin use for the primary prevention of cardiovascular disease and colorectal cancer: U.S. Preventive Services Task Force Recommendation Statement. Ann Intern Med. 2016;164(12):836-845.

50. Veettil SK, Jinatongthai P, Nathisuwan S, et al. Efficacy and safety of chemopreventive agents on colorectal cancer incidence and mortality: systematic review and network meta-analysis. Clin Epidemiol. 2018;10:1433-1445. 
Cancer Management and Research

\section{Publish your work in this journal}

Cancer Management and Research is an international, peer-reviewed open access journal focusing on cancer research and the optimal use of preventative and integrated treatment interventions to achieve improved outcomes, enhanced survival and quality of life for the cancer patient.

The manuscript management system is completely online and includes

Submit your manuscript here: https://www.dovepress.com/cancer-management-and-research-journal 